\title{
A new method to determine the shear coefficient of Timoshenko beam theory
}

\author{
K.T. Chan ${ }^{\mathrm{a}}$, K.F. Lai ${ }^{\mathrm{b}}$, N.G. Stephen ${ }^{\mathrm{c}}$, K. Young ${ }^{\mathrm{b}}$ \\ ${ }^{a}$ Department of Mechanical Engineering, The Hong Kong Polytechnic University, \\ Hong Kong, People's Republic of China \\ ${ }^{b}$ Department of Physics, The Chinese University of Hong Kong, Hong Kong, People's Republic of China \\ ${ }^{c}$ School of Engineering Sciences, Mechanical Engineering, University of Southampton, \\ Highfield, Southampton, SO17 1BJ, UK
}

\begin{abstract}
The frequencies $\omega$ of flexural vibrations in a uniform beam of arbirary cross-section and length $L$ are analyzed by expanding the exact elastodynamics equations in powers of the wavenumber $q=m \pi / L$, where $m$ is the mode number: $\omega^{2}=A_{4} q^{4}+A_{6} q^{6}+\ldots$. The coefficients $A_{4}$ and $A_{6}$ are obtained without further assumptions; the former captures Euler-Bernoulli theory while the latter, when compared with Timoshenko beam theory rendered into the same form, unambiguously yields the shear coefficient $\kappa$ for any cross-section. The result agrees with the consensus best values in the literature, and provides a derivation of $\kappa$ that does not rely on physical assumptions.
\end{abstract}

Keywords: Timoshenko beam, shear coefficient

Email addresses: mmktchan@polyu.edu.hk (K.T. Chan), kflai122@yahoo.com.hk (K.F. Lai), n.g.stephen@soton.ac.uk (N.G. Stephen), kyoung@cuhk.edu.hk (K. Young) 


\section{Nomenclature}

$\begin{array}{ll}A & \text { cross-sectional area, constant } \\ e, E & \text { dilatation, Young's modulus } \\ G & \text { shear modulus } \\ i, j & \text { indices } \\ I & \text { second moment of area } \\ J & \text { integral related to Saint-Venant flexure function } \\ L & \text { length of beam } \\ m & \text { mode number } \\ n & \text { normal, integer, order in } q \\ q & \text { mi/L for a standing wave, wavenumber for a travelling wave } \\ u, v, w & \text { displacement components } \\ x, y, z & \text { Cartesian coordinates } \\ t, T & \text { time, kinetic energy } \\ U & \text { strain energy } \\ \gamma & \text { shear angle } \\ \chi & \text { Saint-Venant flexure function } \\ \kappa & \text { shear coefficient } \\ \lambda & \text { Lamé constant } \\ v & \text { Poisson's ratio } \\ \rho & \text { density } \\ \sigma, \tau & \text { direct, shearing stress } \\ \omega & \text { radian frequency } \\ \psi & \text { cross-sectional rotation }\end{array}$

\section{1. Introduction}

Timoshenko beam theory (TBT) provides shear deformation and rotatory inertia corrections to the classic Euler-Bernoulli theory [1]; it predicts the natural frequency of bending vibrations for long beams with remarkable accuracy if one employs the "best" value for the shear coefficient, $\kappa$. Exact elastodynamic theory is available for beams of circular cross-section (PochammerChree theory, see Love [2], article 202) and the thin (plane stress) rectangular section [3], and for these cases the best coefficients are $\kappa=6(1+v)^{2} /\left(7+12 v+4 v^{2}\right)$ and $\kappa=5(1+v) /(6+5 v)$, respectively, where $v$ is Poisson's ratio of the material. In turn, procedures have been developed for the general cross-section which lead to an expression for the best $\kappa$ in terms of the Saint-Venant flexure function, and which provide the above values when applied to these cross-sections. Stephen and Levinson $[4,5]$ based their methods upon the static stress distribution for a beam subjected to gravity loading, rather than the tip loading assumed in the method proposed by Cowper [6]. More recently, Hutchinson [7] employed the Hellinger-Reissner variational principle to construct a beam theory of Timoshenko type, which incorporated an expression for the shear coefficient that was demonstrated to be equivalent to this best coefficient in the Discussion and Closure section of Ref. [7]. Hutchinson [8] provided further results for thin-walled beams.

Despite these successes, all these works rely on ad hoc physical assumptions and are therefore sometimes queried. It would be of some advantage to be able to dispense with these assumptions, 
insightful though they are, and derive the shear coefficient for an arbitrary cross-section mathematically, and in the process reveal what approximations are in fact employed and therefore what the corrections are. In the present work, we consider standing waves in a beam of length $L$ that is simply-supported (this restriction can be relaxed; see the end of Section 8.1); the governing elastodynamic equations are expanded as a power series in $q=m \pi / L$, the integer $m$ being the mode order; displacements, stress components and frequencies are calculated for each power as necessary. The natural frequency is then expressed, for long thin beams ( $L$ large, $q$ small) as a power series in $q$ :

$$
\omega^{2}=A_{4} q^{4}+A_{6} q^{6}+\ldots
$$

in which symmetry only allows even powers of $q$. (All such series are meant to be asymptotic, not necessarily convergent; this is after all what is needed in applications with a fixed number of terms and $q \rightarrow 0$.) Euler-Bernoulli theory implies that the leading term is $q^{4}$ and in fact gives the value of $A_{4}$. The key in the present discussion is $A_{6}$, and the strategy is to compute it in two different ways and compare the result.

In Section 2, TBT is reviewed, and rendered into the form (1). The resultant $A_{6} \equiv A_{6}^{\mathrm{T}}(\kappa)$ of course depends on $\kappa$.

Then we proceed with an alternate solution, by simply expanding the problem in powers of $q$, without relying on any physical assumptions or introducing any shear coefficient. The governing equations are set up in Section 3 and solved order by order in Section 4. The result is used to evaluate the strain energy $U$ in Section 5 and the kinetic energy $T$ in Section 6 . The eigenvalue $\omega^{2}$ is given by the Rayleigh quotient $Q=U / T$, which is evaluated in Section 7, giving a formula for $A_{6}$ that does not contain $\kappa$. Comparison with $A_{6}^{\mathrm{T}}(\kappa)$ then yields $\kappa$. The key result in (52) turns out to be identical with the canonical expression given by Stephen [4] and Stephen and Levinson [5] for an arbitrary cross-section, thus settling any possible controversy [9] that might remain. A discussion is given in Section 8 and a brief conclusion is given in Section 9.

\section{Timoshenko beam theory}

We consider standing waves in a uniform, isotropic simply-supported beam of arbitrary crosssection and length $L$; the axial coordinate is $z$, and transverse vibration takes place in the $x z$-plane. Euler-Bernoulli theory considers just the transverse displacement $u(z, t)$ and the curvature of the centre line. TBT expresses the centre line slope in terms of the cross-sectional rotation $\psi(z, t)$ and a centre-line shear angle $\gamma(z, t)=\psi(z, t)+\partial u(z, t) / \partial z$; the latter is related to the shear force by a shear coefficient $\kappa$. Within this approximation, the coupled equations of free vibration may be written as

$$
\begin{aligned}
\kappa A G \frac{\partial}{\partial z}\left(\psi+\frac{\partial u}{\partial z}\right) & =\rho A \frac{\partial^{2} u}{\partial t^{2}}, \\
\kappa A G\left(\psi+\frac{\partial u}{\partial z}\right)-E I_{y y} \frac{\partial^{2} \psi}{\partial z^{2}} & =-\rho I_{y y} \frac{\partial^{2} \psi}{\partial t^{2}} .
\end{aligned}
$$

53 Elimination of $\psi$ leads to a single 4th-order differential equation in both space and time 


$$
E I_{y y} \frac{\partial^{4} u}{\partial z^{4}}+\rho A \frac{\partial^{2} u}{\partial t^{2}}-\rho I_{y y}\left(1+\frac{E}{\kappa G}\right) \frac{\partial^{4} u}{\partial z^{2} \partial t^{2}}+\frac{\rho^{2} I_{y y}}{\kappa G} \frac{\partial^{4} u}{\partial t^{4}}=0 .
$$

${ }_{54}$ For a simply-supported beam the mode shape is sinusoidal in both space and time, so write

$$
u \propto \sin q z \sin \omega t,
$$

55 where $q=m \pi / L$ is the wavenumber for mode $m$. The standing wave can be regarded as two 56 superposed travelling waves, and strictly speaking the term "wavenumber" refers to the latter.

57 Equivalently, one can also work with travelling waves, and use a complex notation, e.g. exp [i( $q z_{-}$ $\left.{ }_{58} \omega t\right)$, but for the present paper, the more physical notation of real variables will be used instead.

59 One then has

$$
E I_{y y} q^{4}-\rho A \omega^{2}-\rho I_{y y}\left(1+\frac{E}{\kappa G}\right) q^{2} \omega^{2}+\frac{\rho^{2} I_{y y}}{\kappa G} \omega^{4}=0 .
$$

${ }_{60}$ Now the Euler-Bernoulli frequency $\omega_{\mathrm{EB}}$ is defined by the first two terms in the above, that is,

$$
\omega_{\mathrm{EB}}^{2}=\left(E I_{y y} / \rho A\right) q^{4},
$$

61 and it is convenient to embed this frequency into (5): divide throughout by $E I_{y y} q^{4}$ to give

$$
1-\left(\frac{\omega}{\omega_{\mathrm{EB}}}\right)^{2}-\left(1+\frac{E}{\kappa G}\right) \frac{I_{y y}}{A} q^{2}\left(\frac{\omega}{\omega_{\mathrm{EB}}}\right)^{2}+\frac{E I_{y y}^{2}}{\kappa A^{2} G} q^{4}\left(\frac{\omega}{\omega_{\mathrm{EB}}}\right)^{4}=0 .
$$

For long wavelengths, set all powers of $q$ in (7) equal to zero, which leads to $\omega=\omega_{\mathrm{EB}}$. As the wavelength becomes shorter, that is $q$ becomes larger, so $\left(\omega / \omega_{\mathrm{EB}}\right)$ also becomes less than unity and one ignores the final term in (7), to give

$$
\left(\frac{\omega}{\omega_{\mathrm{EB}}}\right)^{2}=\left[1+\left(1+\frac{E}{\kappa G}\right) \frac{I_{y y}}{A} q^{2}\right]^{-1} .
$$

65 Using (6) for $\omega_{\mathrm{EB}}^{2}$ and employing the binomial expansion on the right-hand side of (8), one gets

$$
\omega^{2}=\frac{E I_{y y}}{\rho A} q^{4}\left[1-\left(1+\frac{E}{\kappa G}\right) \frac{I_{y y}}{A} q^{2}\right]+O\left(q^{8}\right),
$$

66 which gives

$$
\begin{aligned}
A_{4}^{\mathrm{T}} & =\frac{E I_{y y}}{\rho A}, \\
A_{6}^{\mathrm{T}} & =-\frac{E I_{y y}}{\rho A}\left(1+\frac{E}{\kappa G}\right) \frac{I_{y y}}{A},
\end{aligned}
$$

where the superscript T denotes that these come from TBT; $A_{4}^{\mathrm{T}}$ merely expresses classical EulerBernoulli theory, while $A_{6}^{\mathrm{T}}(\kappa)$ will allow $\kappa$ to be determined, if $A_{6}$ can be found in an independent way - which will be the task of the rest of this paper. 


\section{1}

\subsection{Equations of motion}

Assume displacements of the form

$$
\begin{aligned}
u(x, y, z, t) & =\bar{u}(x, y) \sin q z \sin \omega t, \\
v(x, y, z, t) & =\bar{v}(x, y) \sin q z \sin \omega t \\
w(x, y, z, t) & =\bar{w}(x, y) \cos q z \sin \omega t
\end{aligned}
$$

where it is noticed that $(u, v)$ and $w$ are out of phase in $z$ by a quarter cycle. The functions $\bar{u}, \bar{v}$ and $\bar{w}$ are then expanded in powers of $q$. Symmetry implies that $(u, v, w)$ must be odd in $q$, so $\bar{u}$ and $\bar{v}$ are even while $\bar{w}$ is odd in $q$ :

$$
\begin{aligned}
\bar{u} & =u_{0}+u_{2} q^{2}+\ldots \\
\bar{v} & =v_{0}+v_{2} q^{2}+\ldots \\
\bar{w} & =w_{1} q+w_{3} q^{3}+\ldots
\end{aligned}
$$

91

${ }_{92}$ Terms beyond those shown are not necessary for our purpose. The dilatation is likewise expanded

$$
\begin{aligned}
e & \equiv \frac{\partial u}{\partial x}+\frac{\partial v}{\partial y}+\frac{\partial w}{\partial z}=\bar{e}(x, y) \sin q z \sin \omega t \\
\bar{e} & =\left(\frac{\partial u_{0}}{\partial x}+\frac{\partial v_{0}}{\partial y}\right)+\left(\frac{\partial u_{2}}{\partial x}+\frac{\partial v_{2}}{\partial y}-w_{1}\right) q^{2}+\ldots
\end{aligned}
$$

93

94 where $\bar{e}$ is even in $q$.

95 The Navier equations are three of the type 


$$
(\lambda+G) \frac{\partial e}{\partial x}+G \nabla^{2} u=\rho \frac{\partial^{2} u}{\partial t^{2}},
$$

96 where $\nabla^{2}=\partial^{2} / \partial x^{2}+\partial^{2} / \partial y^{2}+\partial^{2} / \partial z^{2}$. Substituting the displacement (12)-(13) and the natural 97 frequency according to (1), one then finds

$$
\begin{aligned}
& (\lambda+G)\left[\frac{\partial^{2}}{\partial x^{2}}\left(u_{0}+u_{2} q^{2}+\ldots\right)+\frac{\partial^{2}}{\partial x \partial y}\left(v_{0}+v_{2} q^{2}+\ldots\right)-\frac{\partial}{\partial x}\left(w_{1} q+\ldots\right) q\right] \\
& +G\left[\left(\frac{\partial^{2}}{\partial x^{2}}+\frac{\partial^{2}}{\partial y^{2}}\right)\left(u_{0}+u_{2} q^{2}+\ldots\right)-\left(u_{0}+u_{2} q^{2}+\ldots\right) q^{2}+\ldots\right] \\
& +\rho\left(u_{0}+u_{2} q^{2}+\ldots\right)\left(A_{4} q^{4}+A_{6} q^{6}+\ldots\right)=0 \\
& (\lambda+G)\left[\frac{\partial^{2}}{\partial x \partial y}\left(u_{0}+u_{2} q^{2}+\ldots\right)+\frac{\partial^{2}}{\partial y^{2}}\left(v_{0}+v_{2} q^{2}+\ldots\right)-\frac{\partial}{\partial y}\left(w_{1} q+\ldots\right) q\right] \\
& +G\left[\left(\frac{\partial^{2}}{\partial x^{2}}+\frac{\partial^{2}}{\partial y^{2}}\right)\left(v_{0}+v_{2} q^{2}+\ldots\right)-\left(v_{0}+v_{2} q^{2}+\ldots\right) q^{2}+\ldots\right] \\
& +\rho\left(v_{0}+v_{2} q^{2}+\ldots\right)\left(A_{4} q^{4}+A_{6} q^{6}+\ldots\right)=0, \\
& (\lambda+G) q\left[\frac{\partial}{\partial x}\left(u_{0}+u_{2} q^{2}+\ldots\right)+\frac{\partial}{\partial y}\left(v_{0}+v_{2} q^{2}+\ldots\right)-\left(w_{1} q+\ldots\right) q\right] \\
& +G\left[\left(\frac{\partial^{2}}{\partial x^{2}}+\frac{\partial^{2}}{\partial y^{2}}\right)\left(w_{1} q+\ldots\right)-\left(w_{1} q+\ldots\right) q^{2}+\ldots\right] \\
& +\rho\left(w_{1} q+\ldots\right)\left(A_{4} q^{4}+A_{6} q^{6}+\ldots\right)=0,
\end{aligned}
$$

99 which are to be solved order by order in $q$; even orders involve only (15a) and (15b), and odd orders only (15c).

\subsection{Boundary conditions}

The boundary conditions of zero traction are

$$
\begin{aligned}
& \sigma_{x} \cos (x, n)+\tau_{x y} \cos (y, n)=0, \\
& \tau_{x y} \cos (x, n)+\sigma_{y} \cos (y, n)=0, \\
& \tau_{x z} \cos (x, n)+\tau_{y z} \cos (y, n)=0,
\end{aligned}
$$

where $\sigma_{i}, i=x, y, z$ are the direct stresses and $\tau_{i j}$ are the shear stresses; they also have a $z$-and $t$-dependence that can be factored out:

$$
\begin{aligned}
\sigma_{i}(x, y, z, t) & =\bar{\sigma}_{i}(x, y) \sin q z \sin \omega t, \quad i=x, y, z, \\
\tau_{x y}(x, y, z, t) & =\bar{\tau}_{x y}(x, y) \sin q z \sin \omega t, \\
\tau_{i z}(x, y, z, t) & =\bar{\tau}_{i z}(x, y) \cos q z \sin \omega t, \quad i=x, y . \\
& 6
\end{aligned}
$$


The direct and shear stress components are given in terms of the strains by

$$
\begin{aligned}
\bar{\sigma}_{x} & =\lambda \bar{e}+2 G(\partial \bar{u} / \partial x), \\
\bar{\sigma}_{y} & =\lambda \bar{e}+2 G(\partial \bar{v} / \partial y), \\
\bar{\sigma}_{z} & =\lambda \bar{e}-2 G \bar{w} q, \\
\bar{\tau}_{x y} & =G(\partial \bar{u} / \partial y+\partial \bar{v} / \partial x), \\
\bar{\tau}_{x z} & =G(\bar{u} q+\partial \bar{w} / \partial x), \\
\bar{\tau}_{y z} & =G(\bar{v} q+\partial \bar{w} / \partial y) .
\end{aligned}
$$

In more detail,

$$
\begin{aligned}
\bar{\sigma}_{x} & =\lambda \bar{e}+2 G\left[\left(\partial u_{0} / \partial x\right)+\left(\partial u_{2} / \partial x\right) q^{2}+\ldots\right] \\
\bar{\sigma}_{y} & =\lambda \bar{e}+2 G\left[\left(\partial v_{0} / \partial y\right)+\left(\partial v_{2} / \partial y\right) q^{2}+\ldots\right] \\
\bar{\sigma}_{z} & =\lambda \bar{e}-2 G\left[w_{1} q^{2}+w_{3} q^{4}+\ldots\right] \\
\bar{\tau}_{x y} & =G\left[\left(\partial u_{0} / \partial y+\partial v_{0} / \partial x\right)+\left(\partial u_{2} / \partial y+\partial v_{2} / \partial x\right) q^{2}+\ldots\right] \\
\bar{\tau}_{x z} & =G\left[\left(u_{0}+\partial w_{1} / \partial x\right) q+\ldots\right] \\
\bar{\tau}_{y z} & =G\left[\left(v_{0}+\partial w_{1} / \partial y\right) q+\ldots\right]
\end{aligned}
$$

108

109 We will also find it convenient to write these components as

$$
\begin{aligned}
\bar{\sigma}_{i} & =\sigma_{i}^{(0)}+\sigma_{i}^{(2)} q^{2}+\ldots, \quad i=x, y, z, \\
\bar{\tau}_{x y} & =\tau_{x y}^{(0)}+\tau_{x y}^{(2)} q^{2}+\ldots, \\
\bar{\tau}_{i z} & =\tau_{i z}^{(1)} q+\tau_{i z}^{(3)} q^{3}+\ldots, \quad i=x, y .
\end{aligned}
$$

in which all terms with the wrong symmetry in $q$ have been dropped. (If these were kept at this point, we would simply find, upon calculation order-by-order, that they in fact vanish.)

\section{Order-by-order solution}

In this Section, we solve the equations of motion (15) subject to the boundary conditions (16), order by order.

\subsection{Zeroth order}

Suppose, as a matter of convention, that the lowest-order displacement is in the $x$-direction, with the normalization set to unity. (Otherwise, all amplitudes will carry a factor $u_{0}$ and all energies a factor $u_{0}^{2}$, which will in the end cancel in $Q=U / T$.) Thus

$$
u_{0}=1, v_{0}=0,
$$

with $w_{0}=0$ already assumed in (12). It is obvious that the equations of motion (15) are satisfied, and since all stress components are zero to this order, the boundary conditions (16) are obviously satisfied as well. 
4.2. First order

The solution is obviously

$$
w_{1}=-x,
$$

with $u_{1}=v_{1}=0$ already assumed in (12). The Navier equations (15) are satisfied, and

$$
\tau_{x z}^{(1)}=G\left(u_{0}+\partial w_{1} / \partial x\right)=0
$$

so that

$$
\nabla^{2} \chi=0
$$

by (21) and (22). All other stress components are obviously zero as well. Again, the boundary conditions need not be considered. For later reference, it is important to note that the stresses therefore start at $O\left(q^{2}\right)$, and hence the strain energy at $O\left(q^{4}\right)$.

\subsection{Second order}

The solution is

$$
u_{2}=v\left(y^{2}-x^{2}\right) / 2, v_{2}=-v x y,
$$

with $w_{2}=0$ already assumed in (12). The only non-zero stress is

$$
\sigma_{z}^{(2)}=E x
$$

This is equivalent to the Euler-Bernoulli stress distribution.

\section{Third order}

For the third order, we only need to determine $w_{3}$, since symmetry dictates $u_{3}=v_{3}=0$. Only (15c) needs to be considered, and this reduces to

$$
\nabla^{2} w_{3}=-2 x
$$

where henceforth and without danger of confusion $\nabla^{2}$ stands for the two-dimension Laplacian: $\nabla^{2}=\partial^{2} / \partial x^{2}+\partial^{2} / \partial y^{2}$. Guided by Love [2], set

$$
w_{3}=-\left(\chi+x y^{2}\right)
$$

To determine the boundary condition on $\chi$, we first note that the two non-zero 3rd-order stresses are 


$$
\begin{aligned}
& \tau_{x z}^{(3)}=G\left(u_{2}+\partial w_{3} / \partial x\right)=-G\left[\partial \chi / \partial x+v x^{2} / 2+(1-v / 2) y^{2}\right] \\
& \tau_{y z}^{(3)}=G\left(v_{2}+\partial w_{3} / \partial y\right)=-G[\partial \chi / \partial y+(2+v) x y]
\end{aligned}
$$

140

141 We only need to consider (16c), which reduces to

$$
\left[\frac{\partial \chi}{\partial x}+\frac{v}{2} x^{2}+\left(1-\frac{v}{2}\right) y^{2}\right] \cos (x, n)+\left[\frac{\partial \chi}{\partial y}+(2+v) y^{2}\right] \cos (y, n)=0
$$

${ }_{142}$ But

$$
\frac{\partial \chi}{\partial x} \cos (x, n)+\frac{\partial \chi}{\partial y} \cos (y, n)=\frac{\partial \chi}{\partial x} \frac{\mathrm{d} x}{\mathrm{~d} n}+\frac{\partial \chi}{\partial y} \frac{\mathrm{d} y}{\mathrm{~d} n}=\frac{\mathrm{d} \chi}{\mathrm{d} n}
$$

so that (30) becomes

$$
\frac{\mathrm{d} \chi}{\mathrm{d} n}=-\left[\frac{v}{2} x^{2}+\left(1-\frac{v}{2}\right) y^{2}\right] \cos (x, n)-(2+v) x y \cos (y, n) .
$$

$$
\begin{aligned}
U=\frac{L}{2} \iint & {\left[\frac{1}{2 E}\left(\bar{\sigma}_{x}^{2}+\bar{\sigma}_{y}^{2}+\bar{\sigma}_{z}^{2}\right)-\frac{v}{E}\left(\bar{\sigma}_{x} \bar{\sigma}_{y}+\bar{\sigma}_{y} \bar{\sigma}_{z}+\bar{\sigma}_{z} \bar{\sigma}_{x}\right)\right.} \\
+ & \left.\frac{1}{2 G}\left(\bar{\tau}_{x y}^{2}+\bar{\tau}_{y z}^{2}+\bar{\tau}_{z x}^{2}\right)\right] \mathrm{d} x \mathrm{~d} y,
\end{aligned}
$$

in which a trivial integration over $z$ has been carried out. Denote

$$
U=U_{0}+U_{2} q^{2}+\ldots
$$




\subsection{Zeroth order}

This will involve terms such as $\tau_{x y}^{(0) 2}$ through to $\sigma_{z}^{(0) 2}$, as well as terms such as $\sigma_{x}^{(0)} \sigma_{y}^{(0)}$, but since all zeroth-order stress components are zero, one immediately has $U_{0}=0$.

\subsection{First order}

This will involve terms such as $2 \tau_{x y}^{(0)} \tau_{x y}^{(1)}$ through to $2 \sigma_{z}^{(0)} \sigma_{z}^{(1)}$, as well as terms such as $\sigma_{x}^{(0)} \sigma_{y}^{(1)}+\sigma_{x}^{(1)} \sigma_{y}^{(0)}$, but since all zeroth-order and first-order stress components are zero, one immediately has $U_{1}=0$.

\subsection{Second order}

This will involve terms such as $\tau_{x y}^{(1) 2}+2 \tau_{x y}^{(0)} \tau_{x y}^{(2)}$ through to $\sigma_{z}^{(1) 2}+2 \sigma_{z}^{(0)} \sigma_{z}^{(2)}$, as well as terms such as $\sigma_{x}^{(0)} \sigma_{y}^{(2)}+\sigma_{x}^{(1)} \sigma_{y}^{(1)}+\sigma_{x}^{(2)} \sigma_{y}^{(0)}$; again all zeroth-order and first-order stress components are zero, and one immediately has $U_{2}=0$.

\subsection{Third order}

This will involve terms such as $2 \tau_{x y}^{(0)} \tau_{x y}^{(3)}+2 \tau_{x y}^{(1)} \tau_{x y}^{(2)}$ through to $2 \sigma_{z}^{(0)} \sigma_{z}^{(3)}+2 \sigma_{z}^{(1)} \sigma_{z}^{(2)}$, as well as terms such as $\sigma_{x}^{(0)} \sigma_{y}^{(3)}+\sigma_{x}^{(1)} \sigma_{y}^{(2)}+\sigma_{x}^{(2)} \sigma_{y}^{(1)}+\sigma_{x}^{(3)} \sigma_{y}^{(0)}$. Again one immediately has $U_{3}=0$.

\subsection{Fourth order}

This will involve terms such as $\tau_{x y}^{(2) 2}+2 \tau_{x y}^{(0)} \tau_{x y}^{(4)}+2 \tau_{x y}^{(1)} \tau_{x y}^{(3)}$ through to $\sigma_{z}^{(2) 2}+2 \sigma_{z}^{(0)} \sigma_{z}^{(4)}+2 \sigma_{z}^{(1)} \sigma_{z}^{(3)}$, as well as terms such as $\sigma_{x}^{(0)} \sigma_{y}^{(4)}+\sigma_{x}^{(1)} \sigma_{y}^{(3)}+\sigma_{x}^{(2)} \sigma_{y}^{(2)}+\sigma_{x}^{(3)} \sigma_{y}^{(1)}+\sigma_{x}^{(4)} \sigma_{y}^{(0)}$. The only non-zero contributor is

$$
\sigma_{z}^{(2)}=E x
$$

and one finds

$$
U_{4}=E I_{y y} L / 4 \text {. }
$$

\subsection{Fifth order}

This involves terms such as $2 \tau_{x y}^{(0)} \tau_{x y}^{(5)}+2 \tau_{x y}^{(1)} \tau_{x y}^{(4)}+2 \tau_{x y}^{(2)} \tau_{x y}^{(3)}$ through to $2 \sigma_{z}^{(0)} \sigma_{z}^{(5)}+2 \sigma_{z}^{(1)} \sigma_{z}^{(4)}+$ $2 \sigma_{z}^{(2)} \sigma_{z}^{(3)}$, as well as terms such as $\sigma_{x}^{(0)} \sigma_{y}^{(5)}+\sigma_{x}^{(1)} \sigma_{y}^{(4)}+\sigma_{x}^{(2)} \sigma_{y}^{(3)}+\sigma_{x}^{(3)} \sigma_{y}^{(2)}+\sigma_{x}^{(4)} \sigma_{y}^{(1)}+\sigma_{x}^{(5)} \sigma_{y}^{(0)}$. The only possible contributors come from the second- and third-order stresses, but since there is no product involving $\sigma_{z}^{(2)}$ and $\tau_{x z}^{(3)}$ or $\tau_{y z}^{(3)}$, one immediately has $U_{5}=0$. This result (and the same for $U_{1}$ and $U_{3}$ ) is anticipated since the strain energy must be even in $q$. 
where $w_{3}$ comes from the last term in (19c) and all reference to eigenfunctions beyond 3rd order has disappeared. One then finds

$$
U_{6}=\frac{E L}{2}\left[J_{1}+\frac{1}{4(1+v)} J_{2}\right] \equiv \frac{E L}{2} J,
$$

\section{. Sixth order}

At this level, there are a variety of terms which do contribute; these are $\tau_{x z}^{(3) 2}$ and $\tau_{y z}^{(3) 2}$, which are straightforward, and also $2 \sigma_{z}^{(4)} \sigma_{z}^{(2)}, \sigma_{x}^{(4)} \sigma_{z}^{(2)}$ and $\sigma_{y}^{(4)} \sigma_{z}^{(2)}$. This suggests that one must determine the direct stress components $\sigma_{i}^{(4)}, i=x, y, z$, but in fact one only needs a knowledge of $w_{3}$. In terms of the displacement components, one has

$$
\begin{aligned}
\sigma_{x}^{(4)} & =\lambda e^{(4)}+2 G \frac{\partial u_{4}}{\partial x}, \\
\sigma_{y}^{(4)} & =\lambda e^{(4)}+2 G \frac{\partial v_{4}}{\partial y}, \\
\sigma_{z}^{(4)} & =\lambda e^{(4)}-2 G w_{3},
\end{aligned}
$$

$$
e^{(4)}=\frac{\partial u_{4}}{\partial x}+\frac{\partial v_{4}}{\partial y}-w_{3}
$$

The relevant expression in the integrand in $U$ is

$$
\frac{1}{2 E}\left(2 \sigma_{z}^{(4)} \sigma_{z}^{(2)}\right)-\frac{v}{E}\left(\sigma_{y}^{(4)} \sigma_{z}^{(2)}+\sigma_{y}^{(2)} \sigma_{z}^{(4)}\right)
$$

which reduces to

$$
-\sigma_{z}^{(2)} w_{3}
$$

in terms of two integrals with dimensions of (length) ${ }^{6}$ defined in terms of the Saint-Venant flexure function:

$$
\begin{aligned}
J_{1}= & \iint x\left(\chi+x y^{2}\right) \mathrm{d} x \mathrm{~d} y, \\
J_{2}= & \iint\left\{\left[\frac{\partial \chi}{\partial x}+\frac{v}{2} x^{2}+\left(1-\frac{v}{2}\right) y^{2}\right]^{2}\right. \\
& \left.+\left[\frac{\partial \chi}{\partial y}+(2+v) x y\right]^{2}\right\} \mathrm{d} x \mathrm{~d} y .
\end{aligned}
$$

194 
203

\section{Kinetic energy}

The Rayleigh quotient $Q$ (see next Section) is essentially the ratio of the strain energy $U$ to the kinetic energy $T$. (This term is used as a shorthand. The kinetic energy is actually $\omega^{2} T$.) Since $U$ starts with $q^{4}$ and we want $Q$ only to $q^{6}$, it suffices to calculate $T$ to just $q^{2}$. Now we have

$$
T=\frac{\rho L}{4} \iint\left(\bar{u}^{2}+\bar{v}^{2}+\bar{w}^{2}\right) \mathrm{d} x \mathrm{~d} y
$$

where again the trivial integration over $z$ has been carried out. The integrand is

$$
\left(u_{0}+u_{2} q^{2}+\ldots\right)^{2}+\left(v_{0}+v_{2} q^{2}+\ldots\right)^{2}+\left(w_{1} q+w_{3} q^{3}\right)^{2} .
$$

The integral of $u_{0}^{2}$ provides the $q^{0}$ term, while $w_{1}^{2}+2 u_{0} u_{2}$ provides the $q^{2}$ term, and one finds

$$
T=T_{0}+T_{2} q^{2}+\ldots
$$

where

$$
\begin{aligned}
T_{0} & =\frac{\rho A L}{4}, \\
T_{2} & =\frac{\rho L}{4}\left[I_{y y}+v\left(I_{x x}-I_{y y}\right)\right] .
\end{aligned}
$$

\section{Rayleigh quotient}

It is well known that the eigenvalue $\omega^{2}$ is given by the Rayleigh quotient [12]

$$
\omega^{2}=Q \equiv \frac{U}{T}=\frac{U_{4} q^{4}+U_{6} q^{6}+\ldots}{T_{0}+T_{2} q^{2}+\ldots}
$$

where $U$ and $T$ are evaluated for the corresponding eigenfunction; from this one finds the coefficients defined by (1) as

$$
\begin{aligned}
& A_{4}=\frac{U_{4}}{T_{0}}=\frac{E I_{y y}}{\rho A} \\
& A_{6}=\frac{U_{6}}{T_{0}}-\frac{U_{4} T_{2}}{T_{0}^{2}}=\frac{E}{\rho A^{2}}\left\{2 A J-I_{y y}\left[I_{y y}+v\left(I_{x x}-I_{y y}\right)\right]\right\}
\end{aligned}
$$

so that compared with (10b), we find 


$$
\kappa=\frac{-2(1+v) I_{y y}^{2}}{2 A J_{1}+A J_{2} /[2(1+v)]+v I_{y y}\left(I_{y y}-I_{x x}\right)} .
$$

Thus we can finally render (49) into

$$
\kappa=\frac{-4(1+v)^{2} I_{y y}^{2}}{2(1+v) A J_{1}+v A J_{3}+2 v(1+v) I_{y y}\left(I_{y y}-I_{x x}\right)} .
$$

This expression is similar but not identical to that given by Hutchinson [7]. But using an identity presented in the Discussion of Ref. [7], we have

$$
J_{2}=v J_{3}-2(1+v) J_{1},
$$

where

$$
\begin{array}{r}
J_{3}=\iint\left\{\left[\frac{x^{2}-y^{2}}{2}\right]\left[\frac{\partial \chi}{\partial x}+\frac{v}{2} x^{2}+\left(1-\frac{v}{2}\right) y^{2}\right]\right. \\
\left.+x y\left[\frac{\partial \chi}{\partial y}+(2+v) x y\right]\right\} \mathrm{d} x \mathrm{~d} y .
\end{array}
$$

In this form, $\kappa$ agrees exactly with the expression for the shear coefficient presented in Ref. [4, 5], thus proving the latter without having to resort to the physical assumption of TBT.

\section{Discussion}

\subsection{General remarks}

Our derivation relies on a single approximation, namely that the wavelength is long, i.e. $q$ is small, so that a power series in $q$ makes sense. There is no need to guess, on physical grounds, what degrees of freedom must be kept. In fact, the new variable $\gamma$ in TBT emerges automatically, in the following way. First, the centre line of the beam tilts by an angle (dropping common time-dependent factors from (11))

$$
\begin{aligned}
\frac{\partial u}{\partial z} & =q \bar{u} \cos q z \\
& =q\left(u_{0}+u_{2} q^{2}+\ldots\right) \cos q z .
\end{aligned}
$$

On the other hand, the cross-sectional plane tilts by an angle

$$
\begin{aligned}
\psi & =\frac{\partial w}{\partial x}=\frac{\partial \bar{w}}{\partial x} \cos q z \\
& =\left(\frac{\partial w_{1}}{\partial x} q+\frac{\partial w_{3}}{\partial x} q^{3}+\ldots\right) \cos q z
\end{aligned}
$$


The centre line and the cross-sectional plane will therefore deviate from orthogonality by an angle

$$
\begin{aligned}
\gamma & =\psi+\frac{\partial u}{\partial z} \\
& =\left[\left(u_{0}+\frac{\partial w_{1}}{\partial x}\right) q+\left(u_{2}+\frac{\partial w_{3}}{\partial x}\right) q^{3}+\ldots\right] \cos q z
\end{aligned}
$$

Our solution, with no assumptions, shows that the first bracket is zero - recovering the key insight and assumption $\gamma=0$ in Euler-Bernoulli theory. The next bracket is not zero, so $\gamma$ must be kept for shorter wavelengths, and moreover its effect is captured once $w_{3}$ is evaluated, without the need to introduce any parameters.

In principle, the expansion in $q$ can be continued and is formally exact, although the evaluation of $u_{4}, v_{4}, w_{5}, \ldots$ is impossible in practice - with two important exceptions to be discussed below. But some general features of the expansion can be noted. First, purely on dimensional grounds, each successive term in (1) has an extra power of $(q a)^{2}$, where $a$ is a typical transverse dimension of the beam, i.e., $A_{2 n} \sim\left(E / \rho a^{2}\right)(q a)^{2 n}$. Second, we do not expect TBT to reproduce the next order exactly, i.e., $A_{8}^{\mathrm{T}} \neq A_{8}$, since for shorter wavelengths, the vibration must be described by more than two variables $u(z, t), \psi(z, t)$ at each $z$ - as can be demonstrated in simple cases (see below). These remarks, taken together, imply that TBT, though highly accurate when $q a \ll 1$, cannot be expected to work when $q a \sim 1$, since the $A_{8} q^{8} \ldots$ terms become important and cannot be reproduced correctly.

The discussion in this paper refers to a simply-supported beam, i.e., hinged-hinged end conditions, but this restriction is unnecessary, since other conditions, e.g., guided-guided or guidedhinged, can be regarded as portions of a multi-span hinged-hinged beam [13].

It should also be mentioned that our "best" choice of $\kappa$ is obtained by matching the $q^{6}$ term in the dispersion relation of the lowest branch (the one without nodes in the cross-sectional plane), and is of course the optimal one for using TBT to describe oscillations of this type - which are the ones most commonly encountered in engineering practice. If one were interested in other types of oscillations, for example the higher branches, other choices may be more appropriate.

\subsection{Solvable examples and possible generalizations}

In two cases, the expansion in $q$ can actually be carried out to very high orders. A brief discussion is given here, principally to illustrate the qualitative remarks above in a precise setting.

First consider flexural vibrations in a hypothetical world of two dimensions, say $x z$. The partial differential equations in Sections 3 and 4 become ordinary differential equations in $x$. Moreover, on dimensional grounds, the $n$th order eigenfunction must go as $(q a)^{n}(x / a)^{k}$, with $k \leq n$ to ensure regularity when $a \rightarrow 0$; thus it must be a polynomial in $x$ of maximum order $n$. The differential equation can be cast into algebraic recursion relations for the polynomial coefficients. With these simplifications, the solution can be carried out to many orders.

Next consider longitudinal vibrations in a circular cylinder. Using cylindrical coordinates $(r, \theta, z)$ and factoring out the trivial $\theta$-dependence, one again obtains an ordinary differential equation (though of a slightly more complicated form) in $r$ and for the same dimensional reason, the solution is again a polynomial in $r$, which likewise can be found to very high orders.

We have solved both of these cases in powers of $q$ to 20 orders. The coefficients in the polynomials turn out to be rational functions of $v$, involving powers up to $v^{3 n-3}$; though complicated, 
these can be obtained using algebraic software packages. If one seeks only numerical values for a specific value of $v$, the computation is much simpler. The results, which can also be checked against exact solutions available in these cases [14] confirm the qualitative features discussed in the last Subsection.

Incidentally, an extension of the two-dimensional problem is a thin plates of thickness $h$, which may be treated in a manner similar to that given in the present paper, to provide a derivation of the "best" value of $\kappa$ for the Mindlin theory of thin plates [15]. That study will be given elsewhere.

\subsection{Using the Rayleigh quotient}

The present paper makes use of the Rayleigh quotient, which has the advantage [10] of giving the 6th-order eigenvalue $A_{6}$ from the 3 rd-order eigenfunction $w_{3}$. Two other nice features should be mentioned as well. First, using any approximate eigenfunction to evaluate $U$ and $T$, and hence $Q$, guarantees positivity. In contrast, the approximation $\omega^{2}=A_{4} q^{4}+A_{6} q^{6}$ goes negative for $q a=O(1)$. Second, again on physical grounds, one expects $\omega^{2} / q^{2} \rightarrow$ constant as $q \rightarrow \infty$ (finite phase velocity). This property is also nicely guaranteed for the Rayleigh quotient, since $U$ involves two extra powers of $q$ compared to $T$. Because of these properties, the Rayleigh quotient is often accurate over a wider range of $q a$. For longitudinal vibrations in a circular cylinder, this method leads to $\sim 5 \%$ accuracy up to $q a \sim 2.5$, namely a cylinder with diameter larger than its length — almost a "disk" rather than a "rod".

\section{Conclusion}

In conclusion, the simple and straightforward strategy of expanding in powers of $q$ provides an alternative method to evaluate the shear coefficient $\kappa$ that is systematic and unambiguous. The expansion, formally exact when carried out to all orders, also provides a wider perspective to view TBT: (a) with the "best" value for $\kappa$, it gives the next $O\left((q a)^{2}\right)$ correction to the classical Euler-Bernoulli theory, but (b) it is itself unlikely to be accurate when $q a \sim 1$.

\section{References}

[1] S.P. Timoshenko, On the transverse vibrations of bars of uniform cross-section. Philosophical Magazine 43 (1922) 125-131.

[2] A.E.H. Love, A Treatise on the Mathematical Theory of Elasticity. Fourth Edition, Dover, New York, 1944.

[3] N.G. Stephen. On "A check on the accuracy of Timoshenko's beam theory". Journal of Sound and Vibration, 257 (2002) 809-812.

[4] N.G. Stephen, Timoshenko's shear coefficient from a beam subjected to gravity loading. ASME Journal of Applied Mechanics, 47 (1980) 121-127.

[5] N.G. Stephen and M. Levinson, A second order beam theory. Journal of Sound and Vibration, 67 (1979) $293-305$.

[6] G.R. Cowper, The shear coefficient in Timoshenko's beam theory. ASME Journal of Applied Mechanics, 33 (1966) 335-340.

[7] J.R. Hutchinson, Shear coefficients for Timoshenko beam theory. ASME Journal of Applied Mechanics, 68 (2001) 87-92. See also Discussion and Closure, ASME Journal of Applied Mechanics, 68 (2001) 959-961.

[8] J.R. Hutchinson, Shear coefficients for thin-walled Timoshenko beams. Third International Symposium on the Vibrations of Continuous Systems, July 2001, Jackson Hole, Wyoming, USA.

[9] J.D. Renton, A check on the accuracy of Timoshenko's beam theory. Journal of Sound and Vibration, 245 (2001) $559-561$.

[10] K.T. Chan, N.G. Stephen and K. Young, Perturbation theory and the Rayleigh quotient. Journal of Sound and Vibration, accepted and in press (Ref: Ms. No. JSV-D-10-00949R1).

[11] S.P. Timoshenko and J.N. Goodier, Theory of Elasticity. Third Edition, McGraw-Hill, 1970. 
308 [12] S.S. Rao, Mechanical Vibrations, Fourth Edition, Pearson Prentice Hall, Upper Saddle River, N.J, U.S.A., 2004.

309 [13] N.G. Stephen. The second spectrum of Timoshenko beam theory — Further assessment. Journal of Sound and $310 \quad$ Vibration, 292 (2006) 372-389.

311 [14] A.E. Armenakis, D.C. Gazis and G. Herrmann, Free Vibration of Circular Cylindrical Shells. Pergamon, Oxford, $312 \quad 1969$.

313 [15] R.D. Mindlin, Influence of rotatory inertia and shear on flexural motions of isotropic elastic plates. ASME Journal 314 of Applied Mechanics 18 (1951) 31-38. 